\title{
Effect of Lepidium meyenii (Maca) on spermatogenesis in male rats acutely exposed to high altitude $(4340 \mathrm{~m})$
}

\author{
G F Gonzales, M Gasco, A Córdova, A Chung, \\ J Rubio and L Villegas
}

Instituto de Investigaciones de la Altura, and Departamento de Ciencias Biológicas y Fisiológicas Facultad de Ciencas y Filosofía, Universidad Peruana Cayetano Heredia, PO Box 1843, Lima, Peru

(Requests for offprints should be addressed to G F Gonzales, Instituto de Investigaciones de la Altura, Universidad Peruana Cayetano Heredia, PO Box 1843, Lima, Peru; Email: iiad@upch.edu.pe)

\begin{abstract}
Lepidium meyenii (Maca) is a Peruvian hypocotyl that grows exclusively between 4000 and $4500 \mathrm{~m}$ in the central Andes. Maca is traditionally employed in the Andean region for its supposed fertility-enhancing properties.

The aim of this study was to test the hypothesis that Maca can prevent high altitude-induced testicular disturbances. Adult male rats were exposed for 21 days to an altitude of $4340 \mathrm{~m}$ and treated with vehicle or aqueous extract of Maca $(666.6 \mathrm{mg} /$ day). The lengths of the stages of the seminiferous epithelium and epididymal sperm counts were obtained at $0,7,14$ and 21 days of exposure. The stages of the seminiferous tubules were assessed by transillumination. A dose-response study was also performed at sea level to determine the effect of Maca given to male rats at doses of $0,6 \cdot 6,66 \cdot 6$ and $666 \cdot 6 \mathrm{mg} /$ day for 7 days on body weight, seminiferous tubule stages and epididymal sperm count. The length of stage VIII and the epididymal sperm count were increased in a dosedependent manner in Maca-treated rats but treatment reduced the length of stage I. At the highest dose, sperm count increased 1.58 times, the length of stage VIII increased 2.4 times and the length of stage I was reduced
\end{abstract}

0.48 times compared with the value at dose 0 . Exposure to high altitude resulted in a reduction in epididymal sperm count after 7 days and lower values were maintained up to 21 days. Altitude reduced spermiation (stage VIII) to half and the onset of spermatogenesis (stages IX-XI) to a quarter on days 7 and 14 but treatment with Maca $(666.6 \mathrm{mg} /$ day) prevented these changes. Data on transillumination and epididymal sperm count in the Macatreated group exposed to high altitude were similar to those obtained at sea level. Maca increased the sperm count on day 21 of exposure to high altitude to values similar $\left(1095 \cdot 25 \pm 20 \cdot 41 \times 10^{6}\right.$ sperm, means \pm S.E.M. $)$ to those obtained in the Maca-treated group at sea level $\left(1132.30 \pm 172.95 \times 10^{6}\right.$ sperm $)$. Furthermore, in the Maca-treated group exposed for 21 days to high altitude, epididymal sperm count was higher than in the nontreated group at sea level $\left(690.49 \pm 43.67 \times 10^{6}\right.$ sperm)

In conclusion, treatment of rats with Maca at high altitude prevented high altitude-induced spermatogenic disruption.

Journal of Endocrinology (2004) 180, 87-95

\section{Introduction}

Being exposed to high altitude means living under conditions of hypoxia, cold, aridity and high ultraviolet radiation. Exposure to high altitude $(4340 \mathrm{~m})$ results in testicular disturbances in men (Donayre 1968) and rats (Gonzales et al. 1990). This was also observed during exposure to a simulated altitude of $4411 \mathrm{~m}$ (Saxena 1995), and also by the effects of cold (Blanco-Rodríguez \& Martinez-Garcia 1997). The changes included degeneration of the germinal epithelium and spermatogenic arrest (Gonzales et al. 1990, Saxena 1995).

Exposure of male rats to high altitude $(4340 \mathrm{~m})$ reduces body weight 3 days after their arrival. This reduction seems also to be due to increased serotonergic activity (Gonzales 1993).

Maca (Lepidium meyenii) is a Peruvian hypocotyl belonging to the Brassicaceae family and grows exclusively between 4000 and $4500 \mathrm{~m}$ altitude in the central Peruvian Andes. The root, known as Maca, has been used traditionally by Peruvians living at high altitudes as a nutrient, an energizer and for aphrodisiac and/or fertilityenhancing properties. According to folk belief, Maca is a plant that enhances fertility in human and domestic animals, which tends to be reduced at higher altitudes (León 1964). The biological activity of the plant is located in the root. The first evidence that Maca improved spermatogenesis was reported in male rats by Gonzales 
et al. (2001a) who found that oral administration to normal adult male rats of an aqueous extract from the roots of $L$. meyenii (Maca) for 14 days had a beneficial effect on spermatogenesis, acting on first mitosis (stages IX-XI). Thereafter, Gonzales et al. (2001b) demonstrated that Maca also improved sperm count and sperm motility in normal men without affecting serum testosterone, luteinizing hormone and follicle-stimulating hormone levels. Its nutritional capability has also been described in mice (Canales et al. 2000).

The present study was designed to determine whether treatment with aqueous extracts of Maca to adult male rats exposed to high altitude may prevent the deleterious effect of altitude on spermatogenesis.

\section{Materials and Methods}

\section{Animals}

Three-month-old male rats of the Holtzman strain obtained from the animal house of the Universidad Peruana Cayetano Heredia were used for the high-altitude exposure study. Rats were divided at random into four groups, two treated and two untreated with Maca. Two groups were maintained at sea level (treated with Maca and untreated) and two groups were kept at high altitude (one treated with Maca and the second untreated). These two groups of rats were transported by bus from Lima at $150 \mathrm{~m}$ to Cerro de Pasco at $4340 \mathrm{~m}$. The trip lasted $8 \mathrm{~h}$. For the dose-response study, 75-day-old rats of the Holtzman strain were used.

Rats were housed four to six per cage, maintained at environmental temperature $\left(22{ }^{\circ} \mathrm{C}\right.$ at sea level and $10{ }^{\circ} \mathrm{C}$ at high altitude) under a $12 \mathrm{~h}$ light: $12 \mathrm{~h}$ darkness cycle and provided with Purina laboratory chow and tap water ad libitum.

\section{Experimental protocols}

Effect of altitude Rats were randomly assigned to four groups, two of them at sea level and two exposed to high altitude $(4340 \mathrm{~m})$. In both locations, one group was treated with Maca $(666.6 \mathrm{mg} /$ day) and the second with vehicle. These four groups was subdivided into three different groups of six animals according to the length of treatment (at $4340 \mathrm{~m}$ ): 7, 14 and 21 days. In the group exposed to high altitude, Maca $(666.6 \mathrm{mg} /$ day $)$ was administered 8-12 h after the mice were received at an altitude of $4340 \mathrm{~m}$.

Dose-response study Four male rats per group received $0,6 \cdot 6,66 \cdot 6$ or $666 \cdot 6 \mathrm{mg}$ aqueous extract of Maca each day for 7 days. The procedure and schedule for administration was similar to that in the altitude experiment. This experiment was performed at sea level.
Treatment To administer Maca or vehicle, an intubation needle no. 18 (Fisher Scientific, Pittsburgh, PN, USA) for nasogastric feeding was used to give, once a day, $2 \mathrm{ml}$ water (with or without Maca) for 7,14 or 21 days. The rats at both locations were killed on days 7, 14 and 21 of treatment by decapitation and blood was collected. The Institutional Review Board of the Scientific Research Office from the Universidad Peruana Cayetano Heredia approved the study.

\section{Preparation of aqueous extract of L. meyenii (Maca)}

The root of L. meyenii was obtained in Carhuamayo at an altitude of $4000 \mathrm{~m}$. The identity of the plant was authenticated by visual verification by Irma Fernandez, a botanist from the Department of Biochemistry, Molecular Biology and Pharmacology, Universidad Peruana Cayetano Heredia. An aqueous extract of the root was prepared according to the traditional method. In brief, $500 \mathrm{~g}$ dried root was placed in a container with $1500 \mathrm{ml}$ water, pulverized and boiled for $30 \mathrm{~min}$. The preparation was left standing to cool and then filtered. The filtrate, containing $333 \mathrm{mg}$ root $/ \mathrm{ml}$ was placed in small vials and kept in a refrigerator at $4{ }^{\circ} \mathrm{C}$ until use.

For the dose-response study, the $333 \mathrm{mg}$ root $/ \mathrm{ml}$ was diluted $1 / 10$ to obtain a concentration of $33 \cdot 3 \mathrm{mg} / \mathrm{ml}$. This was further diluted $1 / 10$ to obtain a concentration of $3.33 \mathrm{mg} / \mathrm{ml}$. Rats received $0,6 \cdot 66,66.6$ or $666.6 \mathrm{mg}$ $\mathrm{Maca} /$ day for 7 days.

\section{Assessment of the stages of the rat seminiferous cycle}

Assessment of the length of the stages was made by transillumination under an inverted stereomicroscope at $40 \times$ magnification as previously described (Gonzales et al. 2001a). A total length of $1000 \mathrm{~mm}$ was assessed for each rat. The stages assessed were as follows: I, II-III, IV-V, VI, VII, VIII, IX-XI, XII and XIII-XIV as described originally by Parvinen (1982).

\section{Epididymal sperm count}

Homogenization-resistant epididymal sperm from nonperfused rats were counted as described previously (Robb et al. 1978) with some modifications. Homogenization was performed in $5 \mathrm{ml}$ saline $(\mathrm{NaCl}, 0.9 \%)$. Modifications included refrigeration of the homogenized epididymal preparation at $4{ }^{\circ} \mathrm{C}$ for $24 \mathrm{~h}$ to allow sperm to be released from the walls. Data are given as sperm/epididymis.

\section{Serum testosterone levels}

Serum testosterone levels were determined by RIA using

${ }^{125}$ I-testosterone as the radioactive marker. The assay was performed using a commercial kit (Diagnostic Products Co, Los Angeles, CA, USA). All samples were run in the 
Table 1 Dose-response effect of Maca on body weight and epididymal sperm count after

7 days treatment. Data are means \pm S.E.M. of four rats per group

\begin{tabular}{|c|c|c|}
\hline & Increase in body weight ( $\mathrm{g}$ ) & Epididymal sperm count $\left(10^{6}\right)$ \\
\hline \multicolumn{3}{|c|}{ Dose of Maca (mg/day) } \\
\hline 0 & $20 \cdot 76 \pm 2 \cdot 35$ & $578 \cdot 91 \pm 2 \cdot 77$ \\
\hline $6 \cdot 66$ & $22 \cdot 50 \pm 0 \cdot 90$ & $618 \cdot 87 \pm 14 \cdot 47^{* \star}$ \\
\hline $66 \cdot 6$ & $35 \cdot 33 \pm 3 \cdot 04^{*}$ & $674 \cdot 26 \pm 2 \cdot 56^{\star *}$ \\
\hline $666 \cdot 6$ & $33 \cdot 25 \pm 3 \cdot 17^{* *}$ & $916 \cdot 57 \pm 7 \cdot 08^{*}$ \\
\hline
\end{tabular}

same assay period. The within-assay variation was $5 \cdot 5 \%$ and sensitivity was $4 \cdot 0 \mathrm{pg} / \mathrm{ml}$.

\section{Statistical analysis}

Data were analyzed using statistical package STATA (version 7.0) for the personal computer (Stata Corporation, College Station, TX, USA). Data are presented as means \pm S.E.M. Homogeneity of variances was assessed by the Bartlett test. If variances were homogeneous, differences between groups over time were assessed by two-way ANOVA. Differences between pairs of means were assessed by the Scheffé test. If variances were not homogeneous, the Kruskal-Wallis test was used to assess differences between groups, and the Mann-Whitney test to assess differences between pairs in each group. Multivariate analysis was performed to assess the independent effects of Maca and altitude on epididymal sperm count. A value of $P<0.05$ was considered to be statistically significant.

\section{Results}

Dose-response study

Table 1 shows data on body weight increase and epididymal sperm count in male rats treated with $0,6 \cdot 6,66 \cdot 6$ or $666.6 \mathrm{mg} \mathrm{Maca} /$ day for 7 days. The increase in body weight from day 0 to day 7 was significantly higher at $66 \cdot 6$ $(P<0 \cdot 01)$ and $666.6 \mathrm{mg} \mathrm{Maca} /$ day $(P<0.05)$ compared with 0 or $6.66 \mathrm{mg} \mathrm{Maca/day.} \mathrm{However,} \mathrm{when} \mathrm{mean} \mathrm{body}$ weight on day 7 was compared no differences were observed between the different doses of Maca $(0,6 \cdot 66$, 66.6, 666.6 mg Maca/day). Epididymal sperm count was significantly increased at all doses of Maca with a significant dose-response effect $\left(r^{2}=0 \cdot 87 ; y=610 \cdot 76+0 \cdot 44 x\right)$. The highest effect on epididymal sperm count was observed at $666.6 \mathrm{mg} \mathrm{Maca/day.}$

Data on the lengths of stages I and VIII of the seminiferous epithelium cycle where a dose-response effect was observed are shown in Fig. 1. The length of stage VIII increased as the dose of Maca increased. In parallel, a reduction in stage I was observed. Stage IX-XI was not modified at any of the doses of Maca $(P$, not significant (NS)).

\section{Maca and exposure to high altitude}

Table 2 shows the body weight changes at 7, 14 and 21 days of exposure. Compared with the untreated group at sea level, body weight was significantly reduced at high altitude up to 14 days $(P<0 \cdot 01)$ and then recovered $(P, \mathrm{NS})$.

Body weight increased in the group treated with Maca at high altitude after $7(P<0 \cdot 01), 14(P<0 \cdot 01)$ and 21 days $(P<0 \cdot 05)$ of exposure; significantly higher than in the untreated group at high altitude.

A reduction in serum testosterone levels on days 14 $(P<0 \cdot 01)$ and $21(P<0 \cdot 01)$ was observed in male rats treated at sea level with vehicle when compared with day 7. This reduction was not observed in the group treated with Maca $(P$, NS) (Table 3).

In the non-treated group at sea level, serum testosterone levels decreased on days $14(P<0 \cdot 01)$ and $21(P<0 \cdot 01)$ compared with values on day 7 . This reduction was not observed in the Maca-treated group ( $P$, NS). However, serum testosterone levels in the Maca-treated group at sea level were higher on day $14(P<0.05)$ than in the untreated group (Table 3). Serum testosterone levels were similar in both non-treated groups at sea level and at high altitude $(P, \mathrm{NS})$. In the untreated group, serum testosterone levels were reduced on day 21 at high altitude $(P<0.05)$ compared with values on day 7 . In the Macatreated group, serum testosterone levels after 7 days at high altitude were higher than in the non-treated group at high altitude (Table 3).

Figure 2 shows data related to the lengths of the stages of the seminiferous tubules measured by transillumination in male rats at sea level and in those exposed acutely at high altitude without or with treatment with Maca on days 7 (Fig. 2a), 14 (Fig. 2b) or 21 (Fig. 2c).

Comparing data between untreated rats at sea level and at high altitude, the lengths of stages VIII and IX-XI were significantly reduced after 7 and 14 days (Fig. 2a and b). 


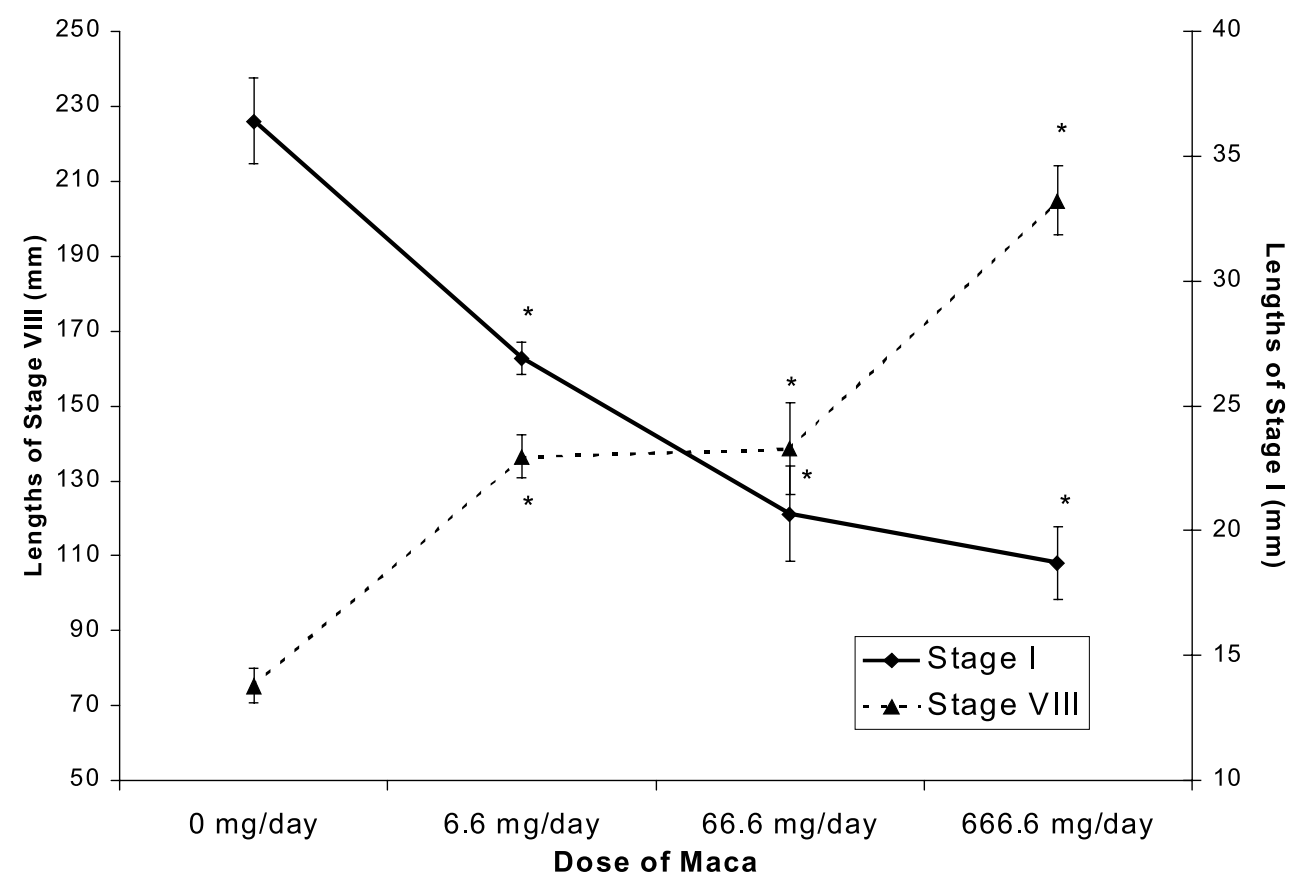

Figure 1 Dose-response curve of the lengths of stages I and VIII of the seminiferous tubules after 7 days of treatment with $0,6 \cdot 6,66 \cdot 6$, or $666 \cdot 6 \mathrm{mg} \mathrm{Maca} /$ day. Data are means \pm S.E.M. ${ }^{*} P<0 \cdot 01$ compared with values at dose 0. Stage I: $r^{2}=0 \cdot 34 ; y=169 \cdot 12-0 \cdot 09 x$. Stage VIII: $r^{2}=0 \cdot 65 ; y=19 \cdot 960 \cdot 02 x$.

At 21 days, untreated rats at high altitude showed stage VIII lengths that were six times higher than at sea level.

At sea level, treatment for 7 days with $666.6 \mathrm{mg}$ $\mathrm{Maca} /$ day resulted in increased lengths of stages IV-VI $(P<0 \cdot 01)$ and VIII $(P<0 \cdot 001)$ with a significant reduction in the length of stage $\mathrm{I}(P<0 \cdot 01)$. The length of stage VIII after treatment with Maca was 6.7 times higher than in the control group. The length of the stages of the onset of spermatogenesis (IX-XI) was not affected by treatment with Maca for 7 days (Fig. 2a). At day 14, Maca treatment significantly increased stages VI $(P<0 \cdot 05)$, VII $(P<0 \cdot 01)$ and IX-XI $(P<0 \cdot 01)$ (Fig. 2b). At day 21, Maca treatment significantly increased stages II-V $(P<0 \cdot 05)$, VIII $(P<0 \cdot 01)$ and XIII-XIV $(P<0 \cdot 01)$ (Fig. $2 c)$.
At high altitude, treatment for 7 days with $666.6 \mathrm{mg}$ $\mathrm{Maca} /$ day resulted in a significant increase in the lengths of stages VI-XII. The highest increase was observed at stage VIII (six times compared with control value). At stage IX-XI, Maca prevented the effect of high altitude. In fact, exposure of untreated rats to high altitude for 7 days resulted in a lower length of stage IX-XI than in Maca-treated rats at high altitude $(P<0 \cdot 01)$ (Fig. 2a). At day 14, Maca treatment significantly increased stages VI, VII, VIII and IX-XI (Fig. 2b). At day 21, treatment significantly increased stages VI and VII $(P<0 \cdot 01)$ (Fig. 2c).

Exposure to high altitude resulted in a reduction in epididymal sperm count at 7,14 and 21 days. This effect

Table 2 Body weight changes in male rats at sea level and during exposure to high altitude (4340 m): effects of Maca. Data are means \pm S.E.M.

\begin{tabular}{|c|c|c|c|c|}
\hline & \multicolumn{2}{|l|}{ Sea level } & \multicolumn{2}{|l|}{ High altitude } \\
\hline & Control & Maca-treated & Control & Maca-treated \\
\hline \multicolumn{5}{|l|}{ Days } \\
\hline $0-7$ & $27 \cdot 17 \pm 1 \cdot 14$ & $25 \cdot 00 \pm 4 \cdot 12$ & $-18 \cdot 89 \pm 4 \cdot 77^{a}$ & $57 \cdot 75 \pm 8 \cdot 56^{*}$ \\
\hline $0-14$ & $52 \cdot 83 \pm 13 \cdot 11$ & $31 \cdot 00 \pm 3 \cdot 13$ & $-37 \cdot 83 \pm 8 \cdot 18^{a}$ & $54 \cdot 40 \pm 7 \cdot 55^{*}$ \\
\hline $0-21$ & $73 \cdot 33 \pm 14 \cdot 91$ & $49 \cdot 25 \pm 7 \cdot 58$ & $40 \cdot 72 \pm 14 \cdot 41$ & $79 \cdot 33 \pm 12 \cdot 74^{* *}$ \\
\hline
\end{tabular}


Table 3 Serum testosterone levels $(\mathrm{ng} / \mathrm{ml})$ in male rats treated or untreated with Maca (666.6 $\mathrm{mg} /$ day) at sea level and during acute exposure to high altitude. Data are means \pm S.E.M. with the number of rats shown in parentheses

\begin{tabular}{|c|c|c|c|c|}
\hline & \multicolumn{2}{|l|}{ Sea level } & \multicolumn{2}{|l|}{ High altitude } \\
\hline & Control & Maca-treated & Control & Maca-treated \\
\hline \multicolumn{5}{|l|}{ Day } \\
\hline 7 & $2 \cdot 13 \pm 0 \cdot 31(10)$ & $2 \cdot 86 \pm 0 \cdot 77(10)$ & $1 \cdot 57 \pm 0 \cdot 46(6)$ & $4 \cdot 60 \pm 1 \cdot 07^{* *}(6)$ \\
\hline 14 & $0 \cdot 92 \pm 0 \cdot 20^{\mathrm{d}}(6)$ & $3 \cdot 36 \pm 0 \cdot 90^{a}(6)$ & $0 \cdot 87 \pm 0 \cdot 46(6)$ & $0 \cdot 78 \pm 0 \cdot 34^{\mathrm{b}, \mathrm{d}}(6)$ \\
\hline 21 & $0 \cdot 88 \pm 0 \cdot 17^{\mathrm{d}}(6)$ & $1 \cdot 77 \pm 0 \cdot 72(6)$ & $0 \cdot 48 \pm 0 \cdot 42^{\mathrm{C}}(6)$ & $1 \cdot 03 \pm 0 \cdot 19^{\mathrm{d}}(6)$ \\
\hline
\end{tabular}

was not observed in male rats treated with Maca (Table 4). At day 21 of exposure to high altitude, epididymal sperm counts in male rats treated with Maca were higher than in untreated rats at sea level $(P<0 \cdot 01)$ and similar to rats treated with Maca at sea level $(P, \mathrm{NS})$.

Table 5 shows multiple regression analysis in which altitude negatively affected epididymal sperm count whereas it was positively affected by treatment with Maca $\left(\mathrm{r}^{2}=0 \cdot 42, P<0 \cdot 0001\right)$.

\section{Discussion}

The present study was aimed at demonstrating whether Maca, as traditionally prepared, might prevent high altitude-induced testicular disturbances. It is known that exposure to high altitude results in damage to spermatogenesis in humans (Donayre 1968) and other animals (Gonzales et al. 1990, Saxena 1995). The present study demonstrated that exposure of male rats to an altitude of $4340 \mathrm{~m}$ for a period of 21 days resulted in low epididymal sperm count. The length of stage VIII was significantly reduced on days 7 and 14 of exposure. This shorter length of stage VIII will result in the lower epididymal sperm count observed on days 7 and 14 of exposure to high altitude.

Spermatogenic arrest induced by high altitude may be due to an increased serotonergic activity (Gonzales et al. 1990). Furthermore, serotonin has been demonstrated to affect spermatogenesis by acting directly on the testis (Hedger et al. 1995), and it has been shown that hypoxia may increase serotonin levels (Awabdy et al. 2003). Serotonin may produce dietary imbalance (Silva et al. 2003). It is known that diet restriction may affect spermatogenesis and sperm number in male rats (Brinkworth et al. 1992). It is therefore possible that dietary imbalance may affect spermatogenesis during exposure to high altitudes.

At a dose of $666.6 \mathrm{mg} /$ day, Maca has been demonstrated to increase spermatogenesis in male rats, acting on stages IX-XI after 14 days of treatment (Gonzales et al. 2001a). In the present study, data were obtained on the effect of Maca on spermatogenesis on days 7, 14 and 21. According to the results of this study, one of the first effects of Maca is on stage VIII where spermiation occurs. In fact, after 7 days of treatment, a significant increase in the length of stage VIII occurred. This increase in the length of stage VIII may explain the increase in epididymal sperm count after 7 days of treatment. The increase in the length of stage VIII was accompanied by a reduction in stage I and an increase in the lengths of stages IV-VI. Final maturation of spermatids occurs from stages I to VII (Kangasniemi et al. 1990) and this may suggest that Maca could act by stimulating the progression of spermatogenesis from stage I to stage VIII where release of spermatozoa to the lumen of the seminiferous tubules occurs. This may increase the amount of spermatozoa in the epididymis as early as day 7 of treatment.

According to our previous report (Gonzales et al. 2001a) on the effect of Maca on spermatogenesis in rats, a dose of $666.6 \mathrm{mg} /$ day in a rat of about $300 \mathrm{~g}$ body weight represents $2 \cdot 2 \mathrm{~g} / \mathrm{kg}$. If we assume that the average weight of a man is $77 \mathrm{~kg}$, this would translate to about $200 \mathrm{~g}$ $\mathrm{Maca} /$ day. This amount would be eaten if a fertilityenhancing property is required.

The dose-response study on the effect of Maca on epididymal sperm count shows that a better effect was observed with a dose of $666.6 \mathrm{mg} / \mathrm{day}(2 \cdot 2 \mathrm{~g} / \mathrm{kg})$. We have previously demonstrated that gelatinized Maca, a pharmaceutical product, at a dose of $1.5-3.0 \mathrm{~g} /$ day for 4 months may improve sperm count in men but this effect was observed in only five out of nine men (Gonzales et al. 2001b). All of this suggests that the doses prescribed by pharmaceutical laboratories to increase sperm count are too low for optimal effect.

The present study demonstrated that Maca $(666.6 \mathrm{mg} /$ day) administered to male rats exposed to high altitude prevented the reduced body weight and epididymal sperm count induced by high altitude. Body weight in the 

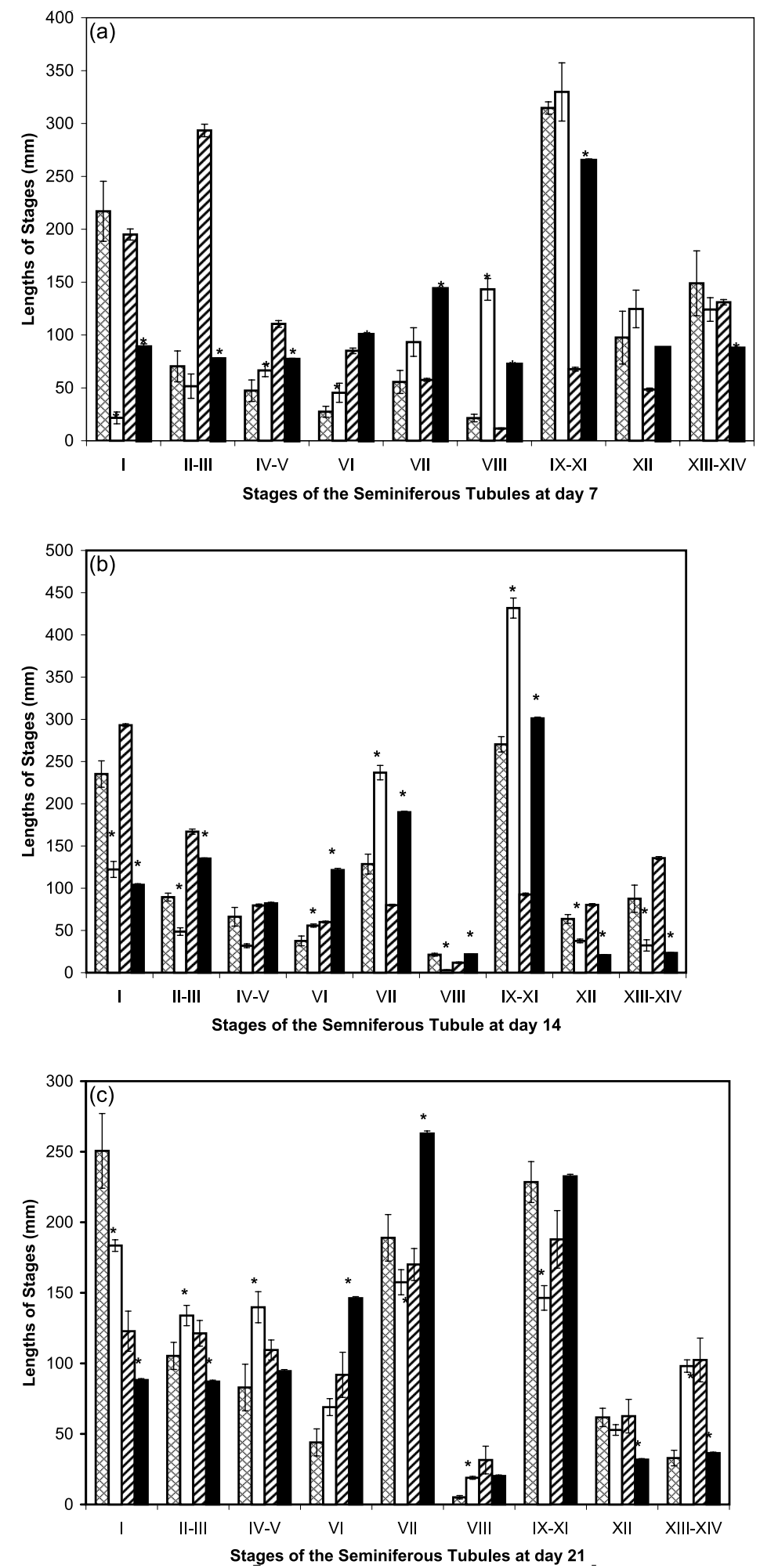

Figure 2 The lengths of stages of the seminiferous tubules on (a) day 7, (b) day 14 and (c) day 21 in untreated male rats at sea level (cross-hatched bars; first bar in each group of four bars), Maca-treated rats at sea level (open bars; second bar in each group), untreated rats at high altitude (hatched bars; third bar) and Maca-treated rats at high altitude (solid bars; fourth bar). Maca was given at a dose of $666.6 \mathrm{mg}$ per day. Data are means \pm S.E.M. ${ }^{\star} P<0 \cdot 01,{ }^{* *} P<0.05$ compared with data from the control group. 
Table 4 Epididymal sperm count $\left(10^{6}\right.$ sperm) in male rats exposed acutely to $4340 \mathrm{~m}$ altitude and either untreated (control group) or treated with an aqueous extract of Maca (Maca-treated group). Data are means \pm S.E.M. with the number of rats shown in parentheses

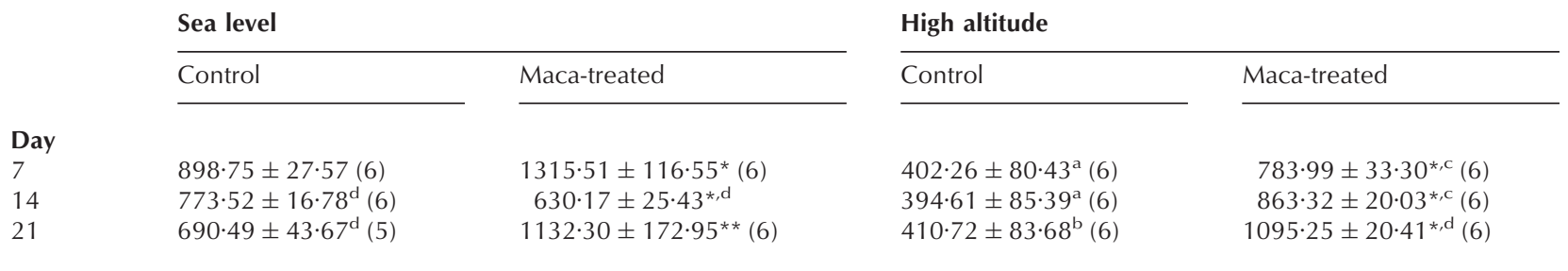

${ }^{*} P<0 \cdot 01,{ }^{*} P<0.05$ compared with control values at high altitude. ${ }^{\mathrm{a} P}<0 \cdot 05,{ }^{\mathrm{b}} P<0.05$ compared with control values at sea level. ${ }^{\mathrm{c}} P<0 \cdot 01$ compared with the Maca-treated group at sea level. ${ }^{\mathrm{d}} P<0 \cdot 01$ compared with values at day 7 .

Maca-treated animals at high altitude was similar to that at sea level, suggesting that Maca had prevented the weight loss. Furthermore, at high altitude, Maca had increased epididymal sperm count on day 21. In fact, male rats treated with Maca and exposed to $4340 \mathrm{~m}$ showed higher lengths of stage VIII (spermiation) and similar lengths of stages IX-XI (onset of spermatogenesis) and similar epididymal sperm counts to those found at sea level, and values significantly higher than those observed in untreated male rats exposed to high altitude. This suggests that Maca reduced the effects of altitude on spermatogenesis by protecting onset (stages IX-XI) and spermiation (stage VIII). The latter avoids the reduction in epididymal sperm count observed on day 7 of exposure.

The effect of Maca is observed at two levels: one of them at stage VIII and the second at stages IX-XI. Stage VIII is associated with spermiation, i.e. the release of spermatozoa to the lumen of the seminiferous tubules. Maca also acts on stages IX-XI where spermatogenesis begins with first mitosis of spermatogonia A (Parvinen 1982). We have previously demonstrated that L. meyenii (Maca) administered for 14 days to normal adult male rats may improve spermatogenesis in spermatogonial mitosis (Gonzales et al. 2001a). Our results demonstrated, in an experimental model in which damage to spermatogenesis was induced by exposure to high altitude, that Maca might also maintain (days 7 and 21 of exposure) and increase the onset of spermatogenesis (day 14). Our data also showed that Maca may act on the stages of spermiation, as stages VII-VIII were significantly increased during exposure to high altitude. The highest value of the length of stage VII was observed on day 21 of exposure to altitude. This may explain the significantly higher value in epididymal sperm count on day 21 of exposure to high altitude than at sea level in male rats treated with Maca.

Treatment for 7 days with Maca $(666.6 \mathrm{mg} /$ day) did not affect serum testosterone levels in male rats. This has also been observed in men treated with 1500 or $3000 \mathrm{mg}$ / day (Gonzales et al. 2003). This suggests that the effect of Maca on spermatogenesis, particularly on stages VIII and IX-XI, could be due to a direct effect on the testis.

Vehicle administered by intubation resulted in the reduction of serum testosterone levels on days 14 and 21 compared with data on day 7 in male rats at sea level. This effect could be due to stressful manipulation with the intubation needle during gastric feeding. Immobilization stress affects testosterone levels in animals (McGrady 1984). The reduction in serum testosterone levels over time was not observed in the group treated with Maca at sea level. Tapia et al. (2000) reported a reduction in stress in mice fed with diets supplemented with Maca, and therefore reducing stress with Maca may prevent the inhibitory effect of intubation on serum testosterone levels. Gonzales et al. (1990) showed that serum testosterone levels in male rats increased after 4 days of exposure to high altitude $(4340 \mathrm{~m})$. Mujica (1994) demonstrated that serum testosterone levels were significantly higher at 7, 14

Table 5 Multiple regression analysis to test the probability that altitude and treatment with Maca affect epididymal sperm count in male rats. Data are means \pm S.E.M.

\begin{tabular}{lcll} 
& Coefficient of regression & & Significance \\
\cline { 2 - 2 } Treatment with Maca & $316.58 \pm 60.97$ & & $<0.0001$ \\
Exposure to altitude & $-327.50 \pm 112 \cdot 01$ & & $<0.005$ \\
Time of exposure to altitude & $79.93 \pm 45 \cdot 73$ & & $<0.087$ \\
Constant & $598.69 \pm 151.66$ & & $<0.0001$
\end{tabular}

Treatment with Maca: yes or no. Exposure to altitude: yes or no. Time of exposure: 0, 7, 14 and 21 days. Coefficient of determination: $r^{2}=0 \cdot 42, P<0 \cdot 0001$. 
and 21 days of exposure to an altitude of $4500 \mathrm{~m}$. In our study at high altitude, we observed that serum testosterone levels were similar to values at sea level on days 7,14 and 21. This may be due to the stress of intubation. Once again, treatment with Maca resulted in higher serum testosterone levels in rats exposed for 7 days to high altitude than in untreated animals.

It is also possible that the stress of transportation and relocation into unfamiliar surroundings may affect serum testosterone levels. In our early observations (Gonzales et al. 1990), control animals at sea level were transported for $10 \mathrm{~h}$ to new surroundings at sea level. In the present study, we have not controlled for transport because of the previous results indicating that altitude per se increases serum testosterone levels. We did not measure serum testosterone levels on day 0 , as the study was designed to compare the effect of altitude on Maca and altitude on days 7, 14, and 21 compared with animals at the same times. However, the results raised the possibility of an effect of stress from the intubation procedure and further study will be required to demonstrate this effect.

It is interesting that altitude affected body weight, spermatogenesis and epididymal sperm count, and that Maca prevented all of these three variables. It is therefore probable that the effect of altitude on spermatogenesis might, in part, be mediated by the reduction in body weight or by a factor that reduces both body weight and spermatogenesis. Serotonin has been implicated as a factor that regulates reduction in both body weight and spermatogenesis at high altitude (Gonzales et al. 1990, Gonzales 1993). However, reduction in body weight per se may affect the reproductive system. In rats, dietary restriction was found to deplete the number of sperm, probably because of a lack of calories and/or non-energetic components of the diet (Brinkworth et al. 1992).

It is possible that Maca may be acting on fertility because of its high nutritional value. The nutritional value of dried Maca hypocotyls is high (Li et al. 2001). Dry Maca hypocotyls have $59 \%$ carbohydrates, $10 \cdot 2 \%$ proteins, $8.5 \%$ fiber, $2 \cdot 2 \%$ lipids and a number of other compounds, including most of the essential amino acids (Dini et al. 1994). Arginine, a constituent of Maca, has been clinically proven to play a role in male fertility (Scibona et al. 1994). Maca also contains sterols, such as campesterol, stigmasterol and $\beta$-sitosterol (Zheng et al. 2000); however $\beta$-sitosterol has been found to be an anti-fertility agent in male rats rather than a compound that enhances fertility (Malini \& Vanithakumari 1991). Piacente et al. (2002) found that Maca also contained (1R,3S)-1methyltetrahydro- $\beta$-carboline- 3 carboxylic acid. $\beta$ Carbolines inhibit apoptosis (Park et al. 2003), and this may be a mechanism that improves spermatogenesis.

Maca is broadly used in Peru as a nutrient. Our data suggest that this nutrient effect needs to be taken into account to explain the fertility-enhancing effect observed at high altitude.

\section{Acknowledgements}

The authors thank Karla Vega, Katiuska Castillo, Rosario Tapia and Sharon Castillo for their help in the fieldwork. Dr Carmen Goñez is acknowledged for her contribution to the radioimmunoassay work. This study was supported by the Vice-rectorate of Investigation, Universidad Peruana Cayetano Heredia.

\section{References}

Awabdy D, Bryan-Lluka LJ \& Wanstall JC 2003

5-Hydroxytryptamine and platelets: uptake and aggregation in hypoxic pulmonary hypertensive rats. European Journal of Pharmacology 459 1-7.

Blanco-Rodrí guez J \& Martinez-Garcia C 1997 Mild hypothermia induces apoptosis in rat testis at specific stages of the seminiferous epithelium. Journal of Andrology 18 535-539.

Brinkworth MH, Anderson D \& McLean AE 1992 Effects of dietary imbalances on spermatogenesis in CD-1 mice and CD rats. Food and Chemical Toxicology 30 29-35.

Canales M, Aguilar J, Prada A, Marcelo A, Huaman C \& Carvajal L 2000 Nutritional evaluation of Lepidium meyenii (MACA) in albino mice and their descendants. Archivo Latinoamericano de Nutrición $\mathbf{5 0}$ 126-133.

Dini A, Migliuolo G, Rastrelli L, Saturnino P \& Schettino O 1994 Chemical composition of Lepidium meyenii. Food Chemistry 49 347-349.

Donayre J 1968 Effect of high altitude on spermatogenesis. Excerpta Medica International Congress 184 1054-1059.

Gonzales GF 1993 High altitude exposure on body weight in male rats: effect of cyproheptadine. Acta Andina 2 169-171.

Gonzales GF, Rodriguez L, Valera J, Sandoval E \& Garcia-Hjarles M 1990 Prevention of high altitude-induced testicular disturbances by previous treatment with cyproheptadine in male rats. Archives of Andrology 24 201-205.

Gonzales GF, Ruiz A, Gonzales C, Villegas L \& Córdova A 2001a Effect of Lepidium meyenii (Maca) roots on spermatogenesis of male rats. Asian Journal of Andrology 3 231-233.

Gonzales GF, Córdova A, Gonzales C, Chung A, Vega K \& Villena A $2001 b$ Improved sperm count after administration of Lepidium meyenii (Maca) in adult men. Asian Journal of Andrology 3 301-304.

Gonzales GF, Córdova A, Vega K, Chung A \&Villena A 2003 Effect of Lepidium meyenii (Maca), a root with aphrodisiac and fertility-enhancing properties, on serum reproductive hormone levels in adult healthy men. Journal of Endocrinology 176 163-168.

Hedger MP, Khatab S, Gonzales GF \& de Kretser DM 1995 Acute and short-term actions of serotonin administration on the pituitary-testicular axis in the adult rat. Reproduction, Fertility and Development 7 1101-1109.

Kangasniemi M, Kaipia A, Mali P, Toppari J, Huhtaniemi I \& Parvinen M 1990 Modulation of basal and FSH-dependent cyclic AMP production in rat seminiferous tubules staged by an improved transillumination technique. Anatomical Record 227 62-76.

León J. 1964 The 'maca' (Lepidium meyenii), a little-known food plant of Peru. Economic Botany 18 122-127.

Li G, Ammermann U \& Quiros CF 2001 Glucosinolate contents in Maca (Lepidium Peruvianum Chacon) seeds, sprouts, mature plants and several derived commercial products. Economic Botany $\mathbf{5 5}$ 255-262.

McGrady AV 1984 Effects of psychological stress on male reproduction: a review. Archives of Andrology 13 1-7.

Malini T \& Vanithakumari G 1991 Antifertility effects of beta-sitosterol in male albino rats. Journal of Ethnopharmacology 35 149-153. 
Mujica E 1994 Acción de la prolactina en los niveles de testosterona plasmática en ratas sometidas a hipoxia de altura. Acta Andina $\mathbf{3}$ $125-136$.

Park TH, Kwon OS, Park SY, Han ES \& Lee CS 2003 N-methylated beta-carbolines protect PC12 cells from cytotoxic effect of MTP+ by attenuation of mitochondrial membrane permeability change. Neuroscience Research 46 349-358.

Parvinen M 1982 Regulation of the seminiferous epithelium. Endocrine Reviews 3 404-417.

Piacente S, Carbone V, Plaza A, Zampelli A \& Pizza C 2002 Investigation of the tuber constituents of maca (Lepidium meyenii Walp). Journal of Agricultural and Food Chemistry 50 5621-5625.

Robb GW, Amann RP \& Killian GJ 1978 Daily sperm production and epididymal sperm reserves of pubertal and adult rats. Journal of Reproduction and Fertility 54 103-107.

Saxena DK 1995 Effect of hypoxia by intermittent altitude exposure on semen characteristics and testicular morphology of male rhesus monkeys. International Journal of Biometeorology 38 137-140.
Scibona M, Meschini P, Capparelli S, Pecori C, Rossi P \& Menchini Fabris GF 1994 L-arginine and male infertility. Minerva Urologica e Nefrologica 46 251-253.

Silva RC, Santos NR \& Brandao ML 2003 Influence of housing conditions on the effects of serotonergic drugs on feeding behavior in non-deprived rats. Neuropsychobiology 47 98-101.

Tapia A, Lopez C, Marcelo A, Canales M \& Aguilar JL 2000 La Maca (Lepidium meyenii) y su efecto anti-stress en un modelo animal en ratones. Acta Andina 8 31-38.

Zheng BL, He K, Kim CH, Rogers L, Shao Y, Huang ZY, Lu Y, Yan SJ, Qien LC \& Zheng QY 2000 Effect of lipidic extract from Lepidium meyenii on sexual behavior in mice and rats. Urology $\mathbf{5 5}$ 598-602.

Received in final form 7 October 2003

Accepted 8 October 2003 